\title{
Stabilization Techniques for the Nonlinear Analytic Nodal Method
}

\author{
Han Gyu Joo, Guobing Jiang, and Thomas J. Downar* \\ Purdue University, School of Nuclear Engineering, 1290 Nuclear Engineering Building \\ West Lafayette, Indiana 47907-1290 \\ Received July 2, 1997 \\ Accepted February 27, 1998
}

\begin{abstract}
The nonlinear analytic nodal method, which is formulated by combining the nonlinear iteration technique and the analytic nodal method (ANM), requires analytic solutions of the two-node problems. When the method is applied to problems that contain near-critical nodes in which there is essentially no net leakage, the two-node ANM solution for such nodes results in highly ill-conditioned matrices and potential numerical instabilities, especially in single precision arithmetic. Two stabilization techniques are introduced to resolve the instability problem by employing alternate basis functions for near-critical nodes. The first uses the exact ANM solution for a critical node, and the second employs the nodal expansion method. Both techniques are shown to perform well; however, the solution accuracy can be mildly sensitive to the criterion used to invoke the stabilized coupling kernel.
\end{abstract}

\section{INTRODUCTION}

Nonlinear nodal methods ${ }^{1}$ have been widely used to solve static and transient reactor physics problems. A wellknown advantage of the nonlinear method is the reduction in computer storage that results from not having to save the expansion coefficients. However, an equally important advantage is a reduction in execution time because the solution variables in the global coarse-mesh finite difference (CMFD) portion of the algorithm are only the node-averaged fluxes for which efficient solution schemes have been developed. Furthermore, the twonode portion of the algorithm lends itself naturally to parallel solution, and when solved in conjunction with a parallel CMFD method, significant execution time reductions can be achieved with the nonlinear nodal method on multiprocessors. ${ }^{2}$

One of principal issues in the implementation of the nonlinear nodal method is the interface current technique used in the two-node coupling relation. As discussed in the review by Lawrence, ${ }^{3}$ two principal classes of transverse-integrated methods have been developed over the years - the polynomial and the analytic methods. In the polynomial nodal expansion method ${ }^{4}$ (NEM), the transverse-integrated equations are solved by approx-

\footnotetext{
*E-mail: downar@ecn.purdue.edu
}

imating the transverse-integrated flux with a truncated polynomial expansion, whereas in the analytic nodal method $^{5}$ (ANM), the analytic solution of the onedimensional neutron diffusion equation is used to solve the transverse-integrated equations. Because the ANM solution to the transverse-integrated equation is exact, it provides a more accurate solution than NEM, particularly when the nodal meshing is large.,

Despite the superior computational accuracy of ANM, most neutronics codes developed during the 1980s employed a two-node coupling based on NEM (Refs. 7, 8, and 9). This was primarily because of the superior computational efficiency of NEM compared with ANM; however there was also some concern about the stability of the ANM solution. ${ }^{10}$ As discussed in Ref. 6 and summarized later in this paper, the linear systems of the ANM two-node solution based on trigonometric and/or hyperbolic functions becomes highly ill-conditioned as the node leakage becomes small, and the nonlinear iteration can fail to converge for computations in single precision arithmetic. The objective of the work reported here was to develop and implement stabilization techniques for the ANM coupling kernel when used in the nonlinear nodal method.

In the following section the derivation of the ANM two-node kernel is presented, and the accuracy and stability of the ANM solution within the nonlinear nodal 
method is analyzed in Sec. III. Stabilization techniques are then introduced in Sec. IV and demonstrated using a modified Nuclear Energy Agency Committee on Reactor Physics (NEACRP) light water reactor (LWR) benchmark problem. Finally, summary and conclusions are provided in Sec. V.

\section{TWO-NODE ANALYTIC NODAL KERNEL}

The distribution of the neutron flux in a reactor can be obtained by solving the multigroup neutron diffusion equation. Since the primary focus of the work here is the LWR, the following development will be simplified by using two energy groups. Using standard notations, the discretized form of the multigroup diffusion equation can be written for node $n$ in terms of the node-average flux $\left(\phi_{g}^{n}\right)$ and the surface-average net currents $\left(J_{g u}^{n \pm}\right)$ :

$$
\sum_{u=x, y, z} \frac{1}{h_{u}^{n}}\left(J_{g u}^{n+}-J_{g u}^{n-}\right)+\sum_{r g}^{n} \phi_{g}^{n}=Q_{g}^{n},
$$

where

$$
Q_{g}^{n}= \begin{cases}\lambda \sum_{g^{\prime}=1}^{2} \nu \Sigma_{f g^{\prime}}^{n} \phi_{g^{\prime}}^{n} & \text { for } g=1 \\ \Sigma_{12}^{n} \phi_{1}^{n} & \text { for } g=2\end{cases}
$$

and $\lambda=1 / k_{\text {eff }}$. The node index $n$ will be omitted for brevity in the following presentation.

In the nonlinear nodal method, the surface-average net current on the interface of any two nodes is represented by the following relation in terms of the nodeaverage fluxes of the left and right nodes: ${ }^{11}$

$$
J_{g}=-D_{g}^{F}\left(\phi_{g}^{r}-\phi_{g}^{l}\right)-D_{g}^{N}\left(\phi_{g}^{r}+\phi_{g}^{l}\right),
$$

where $D_{g}^{F}$ is the base nodal coupling coefficient, determined solely by the finite difference approximation of the neutron current, and $D_{g}^{N}$ is the correctional nodal coupling coefficient, which is determined by the twonode nodal solutions and is updated during the nonlinear iteration. The method used here to update the coupling coefficient will be described in Sec. II.B.

The derivation of nodal kernels for the nonlinear nodal method begins with the transverse-integrated form of the neutron diffusion equation. The static transverseintegrated, one-dimensional, two-group diffusion equations can be written as follows:

$$
-D_{g} \frac{d^{2} \phi_{g}(x)}{d x^{2}}+\Sigma_{r g} \phi_{g}(x)-Q_{g}(x)=-L_{g}(x),
$$

where the transverse leakage source defined as

$$
L_{g}(x) \equiv \frac{1}{h_{y}}\left(J_{g y}^{+}(x)-J_{g y}^{-}(x)\right)+\frac{1}{h_{z}}\left(J_{g z}^{+}(x)-J_{g z}^{-}(x)\right)
$$

is assumed to be known and described by a quadratic polynomial. The transverse-integrated, one-dimensional, twogroup diffusion equations in the $y, z$ directions can be cast in the same form.

The objective of a two-node kernel for the nonlinear nodal method is to find the correctional nodal coupling coefficient $\left(D_{g}^{N}\right)$ to be used in Eq. (3). The current at the interface of every two nodes is expressed in terms of the group constants, the node-average fluxes, the transverse leakages, and the $k_{\text {eff }}$. Estimates of these quantities are provided by the solution of a CMFD problem during the course of the nonlinear iteration and are used to update the transverse leakage source terms and constraints in the two-node problem. Upon convergence of the nonlinear iteration, the interface currents of the CMFD and twonode problems are identical. The following sections present the development of the ANM coupling kernel for a two-node problem in which the $k_{\text {eff }}$, the node-average fluxes, and the quadratic forms of the transverse leakages are provided for each of the two nodes.

\section{II.A. Coupling Kernel Using the Analytic Nodal Method}

Smith used the analytic solution of the transverseintegrated equations as the basis for the ANM (Ref. 5). The general development of the ANM for the threedimensional, corewide implementation is very complicated and is given in Refs. 5 and 12. A much simpler formulation can be derived for a one-dimensional, twonode problem. In the following two subsections, the homogeneous and particular components of the analytic solution are derived for a two-node problem.

\section{II.A.1. Homogeneous Solution}

The analytic solution of the second-order ordinary differential equation given in Eq. (4) consists of a homogeneous and a particular solution. The homogeneous solution is obtained by solving the homogeneous differential equations for which the right side of Eq. (4) is zero. The solution process begins by finding the eigenvalues of the system of the two-group homogeneous differential equations. Let

$$
\frac{d^{2} \phi_{g}^{H}(x)}{d x^{2}}=-B^{2} \phi_{g}^{H}(x) .
$$

This results in a homogeneous linear system

$$
\mathbf{A}\left(B^{2}\right) \phi^{H}(x)=\mathbf{0},
$$

where the $2 \times 2$ matrix is defined as a function of the eigenvalue $B^{2}$ as

$$
\mathbf{A}\left(B^{2}\right) \equiv\left[\begin{array}{cc}
D_{1} B^{2}+\Sigma_{r 1}-\lambda \nu \Sigma_{f 1} & -\lambda \nu \Sigma_{f 2} \\
-\Sigma_{12} & D_{2} B^{2}+\Sigma_{r 2}
\end{array}\right],
$$


and $\phi^{H}(x)$ is a two-element vector consisting of the homogeneous solutions for each group: $\phi^{H}(x) \equiv$ $\left(\phi_{1}^{H}(x), \phi_{2}^{H}(x)\right)^{T}$.

For a nontrivial solution, the determinant of matrix $\mathbf{A}\left(B^{2}\right)$ must be zero; that is,

$$
\left(B^{2}\right)^{2}+\left(\frac{\Sigma_{r 2}}{D_{2}}+\frac{\Sigma_{r 1}}{D_{1}}-\frac{\lambda \nu \Sigma_{f 1}}{D_{1}}\right) B^{2}+\left(\left(1-\lambda k_{\infty}\right) \frac{\Sigma_{r 1}}{D_{1}} \frac{\Sigma_{r 2}}{D_{2}}\right) \equiv\left(B^{2}\right)^{2}+2 b B^{2}+c=0,
$$

where

$$
k_{\infty}=\frac{\nu \Sigma_{f 1}}{\Sigma_{r 1}}+\frac{\Sigma_{12}}{\Sigma_{r 1}} \frac{\nu \Sigma_{f 2}}{\Sigma_{r 2}} .
$$

In the case where the coefficient of the first-order term is positive $(b>0)$, which is true in essentially all the cases of interest in thermal reactor analyses in which the thermal absorption cross section is much larger than the fastfission cross section, the two roots of Eq. (9) for $B^{2}$ are obtained as the following in terms of the constant coefficients appearing in front of the first and zeroth order terms $(b$ and $c)$ :

$$
B_{0}^{2}=-b\left(1-\sqrt{1-\frac{c}{b^{2}}}\right)
$$

and

$$
B_{1}^{2}=-b\left(1+\sqrt{1-\frac{c}{b^{2}}}\right) .
$$

The term inside the square root is always non-negative because it can be rewritten as

$$
\begin{aligned}
b^{2}-c & =\frac{1}{4}\left(\frac{\Sigma_{r 2}}{D_{2}}+\frac{\Sigma_{r 1}}{D_{1}}-\frac{\lambda \nu \Sigma_{f 1}}{D_{1}}\right)^{2}-\left(1-\lambda k_{\infty}\right) \frac{\Sigma_{12}}{D_{1}} \frac{\Sigma_{r 2}}{D_{2}} \\
& =\frac{1}{4}\left(\frac{\Sigma_{r 1}}{D_{1}}-\frac{\lambda \nu \Sigma_{f 1}}{D_{1}}-\frac{\Sigma_{r 2}}{D_{2}}\right)^{2}+\frac{\lambda \nu \Sigma_{f 2}}{D_{2}} \frac{\Sigma_{12}}{D_{1}}>0 .
\end{aligned}
$$

Therefore, the second root, which will be referred to as the first harmonic buckling $B_{1}^{2}$, is a negative real number while the first root, which will be referred to as the fundamental buckling $B_{0}^{2}$, is a real number whose sign depends on the sign of $c$, which is determined by $1-\lambda k_{\infty}$. These two roots are the eigenvalues of the one-dimensional, two-group neutron diffusion equations, Eq. (4). The fundamental eigenvalue determines the asymptotic flux shape realized away from the boundaries of a node, while the first harmonic eigenvalue governs the boundary effects near the boundary. This will become evident with the benchmark problem analyzed in Sec. III.

There are two eigenfunctions for each eigenvalue, which are the solutions of Eq. (6) for the corresponding eigenvalue. They are $\sin / \cos$ or $\sinh / \cosh$ functions, depending on the sign of $B^{2}$. These trigonometric and hyperbolic functions can be represented with two generic functions defined here:

$$
\operatorname{sn}\left(m, \lambda k_{\infty}, x\right) \text { or } c n\left(m, \lambda k_{\infty}, x\right)=\left\{\begin{array}{lll}
\sin (x) & \text { or } \cos (x), & \text { if } m=0 \text { and } \lambda k_{\infty}>1 \\
x & \text { or } 1, & \text { if } m=0 \text { and } \lambda k_{\infty}=1 \\
\sinh (x) & \text { or } \cosh (x), & \text { if } m=1 \text { or } \lambda k_{\infty}<1
\end{array} .\right.
$$

Here the first argument signifies the mode of the eigenvalue. The homogeneous solution is then expressed as the linear combination of the four eigenfunctions; that is,

$$
\phi_{g}^{H}(x)=a_{g 1} \operatorname{sn}\left(0, \lambda k_{\infty}, \kappa x\right)+a_{g 2} \operatorname{cn}\left(0, \lambda k_{\infty}, \kappa x\right)+a_{g 3} \operatorname{sn}\left(1, \lambda k_{\infty}, \mu x\right)+a_{g 4} c n\left(1, \lambda k_{\infty}, \mu x\right),
$$

where

$$
\kappa \equiv \sqrt{\left|B_{0}^{2}\right|} \quad \text { and } \mu \equiv \sqrt{\left|B_{1}^{2}\right|} .
$$

The first two arguments of the eigenfunctions will be omitted in the following because it is possible to make a distinction between different eigenfunctions by $\kappa$ or $\mu$ appearing in the third argument. 
Inserting Eq. (14) into Eq. (4) (with the right side set to zero for the homogeneous solution) yields

$$
\begin{aligned}
& {\left[\mathbf{A}\left(B_{0}^{2}\right)\right]\left[\begin{array}{l}
a_{11} \operatorname{sn}(\kappa x)+a_{12} c n(\kappa x) \\
a_{21} \operatorname{sn}(\kappa x)+a_{22} c n(\kappa x)
\end{array}\right]} \\
& +\left[\mathbf{A}\left(B_{1}^{2}\right)\right]\left[\begin{array}{l}
a_{13} \operatorname{sn}(\mu x)+a_{14} c n(\mu x) \\
a_{23} \operatorname{sn}(\mu x)+a_{24} c n(\mu x)
\end{array}\right]=\left[\begin{array}{l}
0 \\
0
\end{array}\right] .
\end{aligned}
$$

For this equation to hold for all values of $x$, each coefficient of the four eigenfunctions must be zero. Noting this, the fast-to-thermal flux ratios are defined as the following, using the thermal flux equation:

$$
r=\frac{a_{11}}{a_{21}}=\frac{a_{12}}{a_{22}}=\frac{D_{2} B_{0}^{2}+\Sigma_{r 2}}{\Sigma_{12}}
$$

and

$$
s \equiv \frac{a_{13}}{a_{23}}=\frac{a_{14}}{a_{24}}=\frac{D_{2} B_{1}^{2}+\Sigma_{r 2}}{\Sigma_{12}} .
$$

The homogeneous solution can then be compactly written as

$$
\left[\begin{array}{l}
\phi_{1}^{H}(x) \\
\phi_{2}^{H}(x)
\end{array}\right]=\left[\begin{array}{ll}
r & s \\
1 & 1
\end{array}\right]\left[\begin{array}{c}
a_{21} \operatorname{sn}(\kappa x)+a_{22} c n(\kappa x) \\
a_{23} \operatorname{sn}(\mu x)+a_{24} c n(\mu x)
\end{array}\right] .
$$

This equation contains four unknown coefficients per node, which will be determined in Sec. II.A.3 by imposing constraints on node-average fluxes and the continuity of flux and current at the interface of the two-node problem.

\section{II.A.2 Particular Solution}

For a given quadratic transverse leakage approximation applicable for $x$ in $\left[-\left(h_{x} / 2\right),+\left(h_{x} / 2\right)\right]$,

$$
L_{g}(x)=\bar{L}_{g} f_{0}(\xi)+b_{g 1} f_{1}(\xi)+b_{g 2} f_{2}(\xi),
$$

where $\bar{L}_{g}$ is the node-average transverse leakage and

$$
\begin{aligned}
\xi & \equiv \frac{x}{h_{x}}, \quad f_{0}(\xi)=1, f_{1}(\xi)=\xi \\
f_{2}(\xi) & =3 \xi^{2}-\frac{1}{4}
\end{aligned}
$$

The particular solution of the second-order differential equation would have the form

$$
\phi_{g}^{P}(x)=\sum_{p=0}^{4} c_{g p} f_{p}(\xi)
$$

where the third and fourth polynomial basis functions are given by

$$
\begin{aligned}
f_{3}(\xi) & =\xi\left(\xi-\frac{1}{2}\right)\left(\xi+\frac{1}{2}\right), \\
f_{4}(\xi) & =\left(\xi^{2}-\frac{1}{20}\right)\left(\xi-\frac{1}{2}\right)\left(\xi+\frac{1}{2}\right) .
\end{aligned}
$$

Note that these are the basis functions used in the NEM (Ref. 3).

The coefficients used in Eq. (22) are determined by substituting Eq. (22) into Eq. (4) and equating coefficients of the same-order terms. Since the second-order derivatives of the basis functions are

$$
f_{2}^{\prime \prime}(\xi)=6 f_{0}(\xi), \quad f_{3}^{\prime \prime}(\xi)=6 \xi=6 f_{1}(\xi),
$$

and

$$
f_{4}^{\prime \prime}(\xi)=12 \xi^{2}-\frac{3}{5}=4 f_{2}(\xi)+\frac{2}{5} f_{0}(\xi),
$$

inserting Eq. (22) into Eq. (4) yields

$$
\begin{gathered}
-\frac{1}{h_{x}^{2}}\left[\begin{array}{l}
D_{1} g_{1}(\xi) \\
D_{2} g_{2}(\xi)
\end{array}\right]+[\mathbf{A}(0)]\left[\begin{array}{c}
\sum_{p=0}^{4} c_{1 p} f_{p}(\xi) \\
4 \\
\sum_{p=0} c_{2 p} f_{p}(\xi)
\end{array}\right] \\
=-\left[\begin{array}{l}
\bar{L}_{1}+b_{11} f_{1}(\xi)+b_{12} f_{2}(\xi) \\
\bar{L}_{2}+b_{21} f_{1}(\xi)+b_{22} f_{2}(\xi)
\end{array}\right],
\end{gathered}
$$

where

$$
g_{g}(\xi) \equiv 6 c_{g 2}+\frac{2}{5} c_{g 4}+6 c_{g 3} f_{1}(\xi)+4 c_{g 4} f_{2}(\xi) .
$$

Here the $\mathbf{A}(0)$ is the $2 \times 2$ matrix corresponding to a buckling value of zero. It is denoted in the following by $\mathbf{A}_{\infty}$, signifying that it corresponds to zero leakage.

For the equality to hold, each of the five differentorder terms should vanish in the preceding equation. First, the terms containing the third and fourth basis functions should satisfy

$$
\begin{array}{r}
\left.\left[\mathbf{A}_{\infty}\right]\left[\begin{array}{l}
c_{1 p} \\
c_{2 p}
\end{array}\right] \equiv \begin{array}{cc}
\Sigma_{r 1}-\lambda \nu \Sigma_{f 1} & -\lambda \nu \Sigma_{f 2} \\
-\Sigma_{12} & \Sigma_{r 2}
\end{array}\right]\left[\begin{array}{l}
c_{1 p} \\
c_{2 p}
\end{array}\right]=\left[\begin{array}{l}
0 \\
0
\end{array}\right], \\
p=3,4
\end{array}
$$

A nontrivial solution of Eq. (27) exists only when the determinant of the matrix $\mathbf{A}_{\infty}$ is zero, which occurs when $\lambda k_{\infty}=1$ (or $k_{\infty}=k_{\text {eff }}$ ). Otherwise, the solutions of Eq. (27) are zero, yielding vanishing third and fourth coefficients of the particular solution. The most common case $\left(k_{\infty} \neq k_{\text {eff }}\right)$ is first considered in the following subsections, and then the unusual case $\left(k_{\infty}=k_{\text {eff }}\right)$ is discussed.

\section{II.A.2.a. Particular Solution for $k_{\infty} \neq k_{\text {eff }}$}

As discussed earlier, the order of the particular solution in this case is quadratic $\left(c_{g 3}=c_{g 4}=0\right)$. The first 
three coefficients of each group are found by solving the following equations, which follow from the requirement that each order term must vanish:

$$
\begin{aligned}
& {\left[\begin{array}{cc}
\Sigma_{r 1}-\lambda \nu \Sigma_{f 1} & -\lambda \nu \Sigma_{f 2} \\
-\Sigma_{12} & \Sigma_{r 2}
\end{array}\right]\left[\begin{array}{l}
c_{1 p} \\
c_{2 p}
\end{array}\right]=-\left[\begin{array}{l}
b_{1 p} \\
b_{2 p}
\end{array}\right],} \\
& p=0,1,2 \text {, }
\end{aligned}
$$

where $b_{g 1}$ and $\mathrm{b}_{\mathrm{g} 2}$ are given by Eq. (20), and $b_{g 0}$ is a function of $\bar{L}_{g}$ and $D_{g}$ :

$$
b_{g 0}=\bar{L}_{g}-\frac{6}{h_{x}^{2}} D_{g} c_{g 2} .
$$

The solution of Eq. (28) can be expressed as

$$
\mathbf{c}_{p}=-\mathbf{A}_{\infty}^{-1} \mathbf{b}_{p},
$$

in terms of two-element vectors $\mathbf{c}_{p} \equiv\left[c_{1 p}, c_{2 p}\right]^{T}$ and $\mathbf{b}_{p} \equiv\left[b_{1 p}, b_{2 p}\right]^{T}$, and is determined solely by the transverse leakage.

Note that the coefficient matrix $\left(\mathbf{A}_{\infty}\right)$ of Eq. (28) becomes singular when the $k_{\infty}=k_{\text {eff }}$, in which case a unique solution does not exist. In fact, the problem of singularity can be experienced in finite arithmetic computations (especially in single precision) when the nodal $k_{\infty}$ is very close to the core $k_{\text {eff }}$. The determinant of $\mathbf{A}_{\infty}$ will be extremely small in this case, and the system will be illconditioned, leading to a substantial amplification of the transverse leakage in the particular solution. A finite solution is only possible if the large amplification of the particular solution is compensated by the homogeneous solution having the same magnitude but with the opposite sign. However, the compensation may not be correct due to roundoff error, which is nonnegligible in singleprecision arithmetic. This can result in a meaningless solution and, as will be shown in Sec. III.B, can cause divergence of the nonlinear iteration. In the next subsection, the particular solution for $k_{\infty}=k_{\text {eff }}$ is derived such that it can prevent the potential singularity problem.

\section{II.A.2.b. Particular Solution for $k_{\infty}=k_{\text {eff }}$}

With $k_{\infty}=k_{\text {eff }}$, the fundamental-mode buckling of the node is zero, which means there is no net leakage out of this node. All the neutrons coming into the node through the transverse leakage source must leak out through the $x$ direction, and in this sense, the node can be referred to as critical. The derivation of the particular solution for the critical node begins with noting that the two equations in Eq. (4) (one for each group) are no longer linearly independent.

Since the determinant of $\mathbf{A}_{\infty}$ is now zero, the group constants are interrelated; that is, the following ratios are constant $\left(\eta_{2}\right)$ :

$$
\frac{\Sigma_{r 1}-\lambda \nu \Sigma_{f 1}}{\Sigma_{12}}=\frac{\lambda \nu \Sigma_{f 2}}{\Sigma_{r 2}} \equiv \eta_{2}
$$

Multiplying the thermal group equation in Eq. (4) by $\eta_{2}$ and adding it to the fast-group equation results in the simplified equation:

$$
-D_{1} \frac{d^{2} \phi_{1}(x)}{d x^{2}}-\eta_{2} D_{2} \frac{d^{2} \phi_{2}(x)}{d x^{2}}=-L_{1}(x)-\eta_{2} L_{2}(x) .
$$

Integrating Eq. (32) twice results in the following relation between the fast and thermal fluxes:

$$
D_{1} \phi_{1}(x)+\eta_{2} D_{2} \phi_{2}(x)=P_{4}(x),
$$

where

$$
P_{4}(x) \equiv \iint\left(L_{1}(x)+\eta_{2} L_{2}(x)\right) d x d x
$$

or

$$
\begin{gathered}
P_{4}(x)=h_{x}^{2}\left[\frac{1}{4} \hat{b}_{2} f_{4}(\xi)+\frac{1}{6} \hat{b}_{1} f_{3}(\xi)+\left(\frac{1}{6} \hat{b}_{0}-\frac{1}{60} \hat{b}_{2}\right)\right. \\
\left.\times f_{2}(\xi)+C_{1} f_{1}(\xi)+C_{0} f_{0}(\xi)\right]
\end{gathered}
$$

and

$$
\hat{b}_{p} \equiv b_{1 p}+\eta_{2} b_{2 p}, \quad p=0,1,2
$$

and where $C_{0}$ and $C_{1}$ are the integration constants.

Equation (33) can be solved for either the fast or thermal flux. Substituting Eq. (33) into Eq. (4), the following equation is derived for each group flux:

$$
-\frac{d^{2} \phi_{g}(x)}{d x^{2}}+2 b \phi_{g}(x)=\Gamma_{g} P_{4}(x)-\frac{L_{g}(x)}{D_{g}},
$$

where

$$
\Gamma_{g} \equiv \frac{1}{D_{1} D_{2}} \cdot\left\{\begin{array}{ll}
\Sigma_{r 2}, & g=1 \\
\Sigma_{12}, & g=2
\end{array} .\right.
$$

Note here that the equality in Eq. (31) was used to obtain the second term on the left side. This equation now can be used to determine the particular solution. By inserting the quartic polynomial given in Eq. (22) into Eq. (36), the five coefficients in the particular solution for the thermal group can be determined as a function of the two integration constants, $C_{0}$ and $C_{1}$, by equating coefficients for each order term of the left and right sides. The complete set of coefficients are given as follows: 
$c_{g p}=\frac{1}{2 b}$

$$
\times \begin{cases}h_{x}^{2} \Gamma_{g} \frac{\hat{b}_{2}}{4}, & p=4 \\ h_{x}^{2} \Gamma_{g} \frac{\hat{b}_{1}}{6}, & p=3 \\ h_{x}^{2} \Gamma_{g}\left(\frac{\hat{b}_{0}}{6}-\frac{\hat{b}_{2}}{60}\right)+\frac{4 c_{g 4}}{h_{x}^{2}}-\frac{b_{g 2}}{D_{g}}, & p=2 . \\ h_{x}^{2} \Gamma_{g} C_{1}+\frac{6 c_{g 3}}{h_{x}^{2}}-\frac{b_{g 1}}{D_{g}}, & p=1 \\ h_{x}^{2} \Gamma_{g} C_{0}+\frac{1}{h_{x}^{2}}\left(\frac{2}{5} c_{g 4}+6 c_{g 2}\right)-\frac{\bar{L}_{g}}{D_{g}}, & p=0\end{cases}
$$

The zeroth and first coefficients given in Eq. (38) are not unique because they contain one arbitrary integration constant, $C_{0}$ and $C_{1}$, respectively. Noting that in the critical node case, the zeroth and the first-order terms are one part of the homogeneous solutions as shown in Eq. (13) for $\lambda k_{\infty}=1$, it is possible to combine the terms containing $C_{0}$ and $C_{1}$ with the homogeneous solution. This means that the two integration constants can be chosen to be zero, that is, $C_{0}=C_{1}=0$, so that the particular solution is now uniquely determined.

\section{II.A.3. Final Solution}

The final solution of Eq. (4) is the summation of the homogeneous and particular solutions, $\phi(x)=\phi^{H}(x)+$ $\phi^{P}(x)$, and can now be written as

$$
\begin{aligned}
{\left[\begin{array}{l}
\phi_{1}(x) \\
\phi_{2}(x)
\end{array}\right]=} & {\left[\begin{array}{l}
\phi_{1}^{H}(x) \\
\phi_{2}^{H}(x)
\end{array}\right]+\left[\begin{array}{l}
\phi_{1}^{P}(x) \\
\phi_{2}^{P}(x)
\end{array}\right] } \\
= & {\left[\begin{array}{ll}
r & s \\
1 & 1
\end{array}\right]\left[\begin{array}{c}
a_{21} s n(\kappa x)+a_{22} c n(\kappa x) \\
a_{23} s n(\mu x)+a_{24} c n(\mu x)
\end{array}\right] } \\
& +\left[\begin{array}{c}
\sum_{p=0}^{4} c_{1 p} f_{p}(\xi) \\
\sum_{p=0}^{4} c_{2 p} f_{p}(\xi)
\end{array}\right] .
\end{aligned}
$$

So far, the two components of the analytic solution were considered only for one of the two nodes in the twonode problem. As shown in the previous subsections, the particular solution is determined solely by the transverse leakage for the node of interest. Information from the neighboring node is not required. The four undetermined coefficients in the homogeneous solution in Eq. (39), however, must be determined such that they satisfy constraints on the continuity of flux and current at the interface of the two nodes.

Since there are four unknown coefficients per node, there are a total of eight coefficients to be determined in a two-node problem. These eight coefficients are uniquely determined by applying the following eight constraints: four node-average flux constraints ( 2 groups $\times 2$ nodes), two flux continuity ( 2 groups $\times 1$ interface), and two current continuity ( 2 groups $\times 1$ interface). In the following, the determination of these eight unknown coefficients is presented.

The node-average flux constraints can be written

$$
\begin{gathered}
\bar{\phi}_{g}^{n}=\frac{1}{h_{x}^{n}} \int_{-h_{x}^{n} / 2}^{h_{x}^{n} / 2} \phi_{g}^{n}(x) d x=\int_{-1 / 2}^{1 / 2} \phi_{g}^{n}\left(h_{x}^{n} \xi\right) d \xi, \\
n=r \text { or } l,
\end{gathered}
$$

with $l$ and $r$ designating the left and right node of the interface, respectively. This results in the following equation for each node $n$ :

$$
\begin{gathered}
{\left[\begin{array}{cc}
r_{n} & s_{n} \\
1 & 1
\end{array}\right]\left[\begin{array}{cc}
\frac{s n\left(\kappa_{1 / 2}^{n}\right)}{\kappa_{1 / 2}^{n}} & 0 \\
0 & \frac{s n\left(\mu_{1 / 2}^{n}\right)}{\mu_{1 / 2}^{n}}
\end{array}\right]\left[\begin{array}{l}
a_{22}^{n} \\
a_{24}^{n}
\end{array}\right]} \\
=\left[\begin{array}{c}
\bar{\phi}_{1}^{n} \\
\bar{\phi}_{2}^{n}
\end{array}\right]-\left[\begin{array}{c}
c_{10}^{n} \\
c_{20}^{n}
\end{array}\right],
\end{gathered}
$$

where

$$
\kappa_{1 / 2}^{n} \equiv \kappa_{n} \frac{h_{x}^{n}}{2} \quad \text { and } \quad \mu_{1 / 2}^{n} \equiv \mu_{n} \frac{h_{x}^{n}}{2} .
$$

The two even coefficients of each node can then be determined by solving the preceding two $2 \times 2$ equations. Note that no information is required from the neighboring node.

The odd coefficients are now solved by applying the constraints of continuity of flux and net current at the interface, which read

$$
\zeta_{g}^{l} \phi_{g}^{l}\left(\frac{h_{x}^{l}}{2}\right)=\zeta_{g}^{r} \phi_{g}^{r}\left(-\frac{h_{x}^{r}}{2}\right)
$$

and

$$
-\left.D_{g}^{l} \frac{d \phi_{g}^{l}(x)}{d x}\right|_{h_{x}^{l} / 2}=-\left.D_{g}^{r} \frac{d \phi_{g}^{r}(x)}{d x}\right|_{-h_{x}^{r} / 2},
$$

where $\zeta_{g}^{n}$ is the discontinuity factor.

Substituting Eq. (39) into Eqs. (43) and (44), a $4 \times 4$ equation can be obtained as follows:

$$
\begin{aligned}
{\left[\begin{array}{cc}
\boldsymbol{\Phi}_{l}(s n) & \boldsymbol{\Phi}_{r}(s n) \\
-\mathbf{J}_{l}\left(s n^{\prime}\right) & \mathbf{J}_{r}\left(s n^{\prime}\right)
\end{array}\right]\left[\begin{array}{l}
\mathbf{a}_{o}^{l} \\
\mathbf{a}_{o}^{r}
\end{array}\right]=} & {\left[\begin{array}{cc}
-\boldsymbol{\Phi}_{l}(c n) & \boldsymbol{\Phi}_{r}(c n) \\
\mathbf{J}^{l}\left(c n^{\prime}\right) & \mathbf{J}^{r}\left(c n^{\prime}\right)
\end{array}\right]\left[\begin{array}{c}
\mathbf{a}_{e}^{l} \\
\mathbf{a}_{e}^{r}
\end{array}\right] } \\
& +\left[\begin{array}{c}
\phi_{r}^{P}-\phi_{l}^{P} \\
\mathbf{J}_{r}^{P}-\mathbf{J}_{l}^{P}
\end{array}\right]
\end{aligned}
$$


where the $2 \times 2$ matrices $\boldsymbol{\Phi}_{n}$ and $\mathbf{J}_{n}$ are defined as the following functional of $f$ :

$$
\boldsymbol{\Phi}_{n}(f) \equiv\left[\begin{array}{cc}
\zeta_{1}^{n} r_{n} f\left(\kappa_{1 / 2}^{n}\right) & \zeta_{1}^{n} s_{n} f\left(\mu_{1 / 2}^{n}\right) \\
\zeta_{2}^{n} f\left(\kappa_{1 / 2}^{n}\right) & \zeta_{2}^{n} f\left(\mu_{1 / 2}^{n}\right)
\end{array}\right]
$$

and

$$
\mathbf{J}_{n}(f) \equiv\left[\begin{array}{cc}
D_{1}^{n} r_{n} \kappa_{n} f\left(\kappa_{1 / 2}^{n}\right) & D_{1}^{n} s_{n} \mu_{n} f\left(\mu_{1 / 2}^{n}\right) \\
D_{2}^{n} \kappa_{n} f\left(\kappa_{1 / 2}^{n}\right) & D_{2}^{n} \mu_{n} f\left(\mu_{1 / 2}^{n}\right)
\end{array}\right] ;
$$

the two element vectors consisting of the set of odd and even coefficients are defined as

$$
\mathbf{a}_{o}^{n} \equiv\left[a_{21}^{n}, a_{23}^{n}\right]^{T} \text { and } \mathbf{a}_{e}^{n} \equiv\left[a_{22}^{n}, a_{24}^{n}\right]^{T} ;
$$

the two element vectors for contributions from the particular solution are defined as

$$
\phi_{n}^{P} \equiv\left[\zeta_{1}^{n} \phi_{1}^{P n}, \zeta_{2}^{n} \phi_{2}^{P n}\right]^{T} \text { and } \mathbf{J}_{n}^{P} \equiv\left[J_{1}^{P n}, J_{2}^{P n}\right]^{T}
$$

and the scalars are defined as

$$
\begin{aligned}
& \phi_{g}^{P n} \equiv c_{g 0}^{n}+(-1)^{i} \frac{c_{g 1}^{n}}{2}+\frac{c_{g 2}^{n}}{2} \\
& J_{g}^{P n}=-\frac{D_{g}^{n}}{h_{x}^{n}}\left(c_{g 1}^{n}+(-1)^{i} 3 c_{g 2}^{n}+\frac{c_{g 3}^{n}}{2}+(-1)^{i} \frac{c_{g 4}^{n}}{5}\right),
\end{aligned}
$$

with the exponent $i$ being defined as follows:

$$
i=\left\{\begin{array}{ll}
0 & \text { if } n=l \\
1 & \text { if } n=r
\end{array} .\right.
$$

The $s n^{\prime}$ and $c n^{\prime}$ used in Eq. (45) are the derivatives of the corresponding functions defined in Eq. (13). The solution of Eq. (45) now determines the odd function coefficients of the two nodes.

With the even and odd coefficients determined by solving Eqs. (41) and (45), respectively, the eight unknown coefficients in the two-node problem are now fully determined, and the fast and thermal fluxes given in Eq. (39) are known. Substituting these fluxes into the right or left side of Eq. (44), the net nodal current at the interface of the two nodes can be calculated. The correction term coefficient $D_{g}^{N}$ can then be obtained according to Eq. (3), completing the two-node ANM kernel calculation.

\section{II.B. Implementation of the Analytic Nodal Method Kernel Within the Nonlinear Nodal Method}

In a nonlinear nodal calculation, there are two major problems to be solved: a CMFD problem and a set of two-node problems. They are solved alternately, with each providing the input for the other problem. The solution of a CMFD problem provides the node-average flux dis- tribution to be used in the subsequent two-node problems, and the solution of the two-node problems provides the correctional nodal coupling coefficients to be used to formulate a new CMFD problem. There are numerous methods for solving the CMFD problem, which involves a fission source iteration for the solution of an eigenvalue problem as well as the solution of a sparse linear system. In the work here, a Krylov subspace method ${ }^{2}$ was employed to solve the linear system appearing in the fission source iteration, and the Wielandt shift method ${ }^{13}$ was used for the fission source acceleration.

Since the flux distribution as well as the corrective nodal coupling coefficients will not be fully converged until the global nonlinear iteration converges, only partial convergence was achieved for each CMFD problem by updating the corrective nodal coefficients after a fixed number of fission source iterations (usually three to five iterations). The two-node ANM kernel derived in the previous section was implemented into a spatial kinetics PARCS $\operatorname{code}^{14}$ using the nonlinear iteration scheme described earlier. The PARCS code also has the two-node NEM kernel.

\section{ACCURACY AND STABILITY OF THE TWO-NODE ANALYTIC NODAL METHOD KERNEL}

To verify the accuracy of the two-node ANM kernels, a series of benchmark problems, including the NEACRP pressurized water reactor (PWR) rod ejection problems, ${ }^{15}$ were analyzed using PARCS. Since it was possible to extend the two-node ANM kernel to the solution of a transient fixed-source problem with the minor additional effort of combining the effective source term into the transverse leakage term, the two-node ANM kernel was verified for the transient problems as well as the eigenvalue problems. In this section, a detailed analysis of the accuracy and stability of the two-node ANM kernel is presented only for a set of eigenvalue problems. The performance of the two-node ANM kernel for the transient problems is reported elsewhere. ${ }^{6,16}$ In the following subsection, the accuracy of the ANM solution will be demonstrated using the NEACRP PWR spatial kinetics benchmark case A1 problem. The stability of the ANM solution is then analyzed for a slightly modified version of the A1 core configuration such that it introduces nearcritical nodes.

\section{III.A. Analysis of the Initial State of Nuclear Energy Agency Committee on Reactor Physics Case Al}

The NEACRPPWR spatial kinetics problem is a threedimensional benchmark that has been widely analyzed by modern nodal codes. The core consists of 157 fuel assemblies and is heavily rodded in all the benchmark cases. Among the six cases, case A1 corresponds to a hot-zeropower (HZP) condition in which a large number of rods 


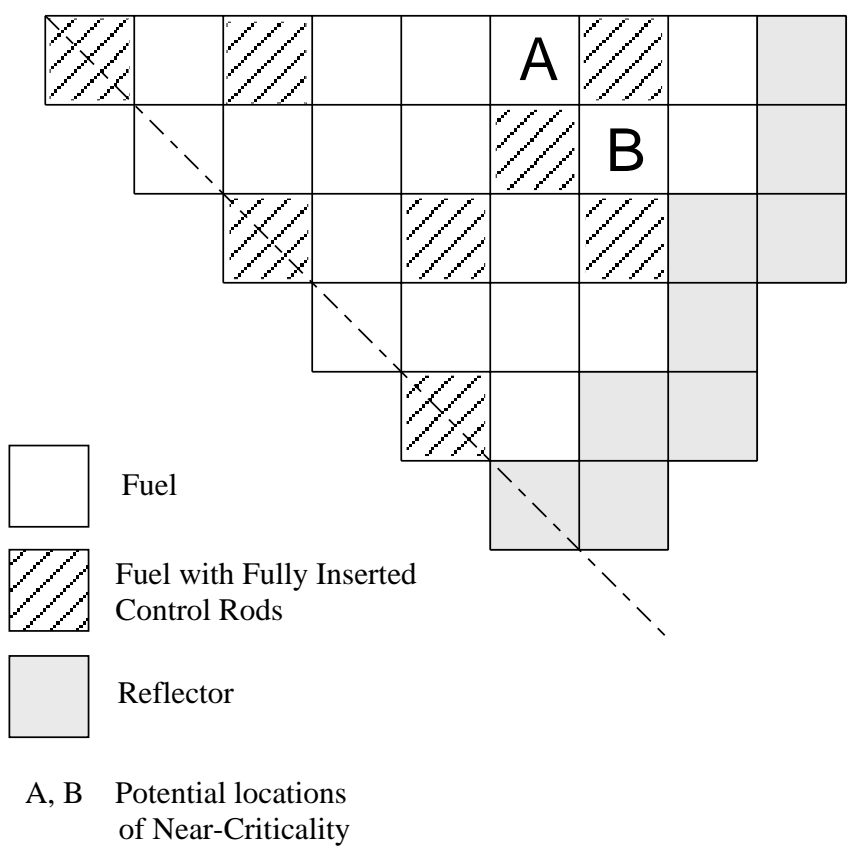

Fig. 1. Core configuration of the NEACRP benchmark problem case A1.

are inserted causing a very nonuniform radial power distribution (maximum assembly relative power $F x y=$ 1.91). The core configuration is shown in Fig. 1.

The initial state of this problem was analyzed using the ANM kernel in PARCS for several radial node sizes, keeping the axial mesh nodalization constant as given in the specifications (18 planes). Corresponding NEM calculations were also performed for purposes of compari- son. The number of nodes examined were 1,4 , and 16 nodes/fuel assembly (FA). The calculations were performed on a Sun UltraSparc II workstation with doubleprecision arithmetic. The results are summarized in Table I in terms of the errors in the computed eigenvalue and in the relative power density (RPD). Here the reference was taken from the 16 node/FA ANM results, and the boron concentration of $561.3 \mathrm{ppm}$ corresponds to the critical boron concentration (CBC) for the ANM 4 node/ FA case. This agrees extremely well with the reference calculation recently performed by PANTHER for which the $\mathrm{CBC}$ is $561.2 \mathrm{ppm}$ (Ref. 17).

As shown in Table I, both ANM and NEM provide essentially the same solution with a very fine spatial mesh (that is, 16 nodes/assembly); however, ANM provides a more accurate solution than NEM at the coarser mesh of 1 and 4 nodes/FA, which are typically used in reactor analysis. The maximum RPD error of NEM is $\sim 2 \%$ for 4 nodes/assembly and occurs at the periphery of the core. With 4 nodes/FA, there is a global tilt in the NEM solution such that the flux in the peripheral region is overpredicted and the flux in the central region is underpredicted. This is primarily because the polynomial basis function used in NEM can potentially cause large errors in the flux prediction at the fuel-reflector interface. Although errors in the NEM solution are not large, the misprediction of the flux distribution can significantly impact the prediction of core transient behavior for events such as a control rod ejection from HZP conditions, which can cause a super-prompt critical transient. ${ }^{16}$

Although the accuracy of the ANM solution is very good for the 4 node/FAcase and is always superior to NEM, the errors of ANM for 1 node/FA are somewhat large and can be attributed to the quadratic transverse leakage approximation, which becomes less valid as the node size

TABLE I

Comparison of the NEM/ANM Eigenvalue Calculation Results for the NEACRP Case A1

\begin{tabular}{|c|c|c|c|c|c|}
\hline Node/FA & \multicolumn{2}{|c|}{1} & \multicolumn{2}{|c|}{4} & 16 \\
\hline Kernel & NEM & ANM & NEM & ANM & NEM \\
\hline $\begin{array}{l}k_{\text {eff }} \text { error }^{\mathrm{a}}\left(\mathrm{pcm}, \times 10^{-5}\right) \\
\text { Positive RPD error }(\%) \\
\text { Negative RPD error }(\%)\end{array}$ & $\begin{array}{r}83 \\
-3.88 \\
9.07\end{array}$ & $\begin{array}{l}43 \\
-2.02 \\
4.25\end{array}$ & $\begin{array}{r}14 \\
-0.72 \\
1.96\end{array}$ & $\begin{array}{r}3 \\
-0.16 \\
0.40\end{array}$ & $\begin{array}{rl}1 & 1 \\
-0.07 \\
0.23\end{array}$ \\
\hline CPU Time ${ }^{\mathrm{b}}, \mathrm{s}$ & \multicolumn{2}{|c|}{ NEM } & \multicolumn{2}{|c|}{ ANM } & Ratio \\
\hline $\begin{array}{l}\text { Nodal calculation only } \\
\text { Total }\end{array}$ & \multicolumn{2}{|c|}{$\begin{array}{l}1.73 \\
4.41\end{array}$} & \multicolumn{2}{|c|}{$\begin{array}{l}2.09 \\
4.78\end{array}$} & $\begin{array}{l}1.21 \\
1.08\end{array}$ \\
\hline
\end{tabular}

${ }^{\mathrm{a}}$ The 16 node/FA case is taken as the reference. The reference $k_{\text {eff }}$ is 0.99996.

${ }^{\mathrm{b}}$ For 4 node/FA calculations only. 
increases or the flux distribution has stronger gradients. The CPU time comparison shown in Table I indicates that the ANM kernel requires $\sim 20 \%$ more computation time than the NEM kernel in the two-node nodal calculations. This is primarily because of the additional computation time necessary to evaluate the trigonometric and hyperbolic functions used in the ANM calculations. However, the total computation time increases by only $8 \%$ because the nodal calculation requires only $\sim 40 \%$ of the total computation time.

\section{III.B. Modified Nuclear Energy Agency Committee on Reactor Physics Problem and Numerical Instability}

In the benchmark problem analyzed in the previous subsection, there was no case in which the nodal $k_{\infty}$ was close enough to the core $k_{\text {eff }}$ to cause an instability problem, primarily because the flux distribution was relatively heterogeneous and there was substantial leakage in all the nodes. To investigate the stability of the ANM solution for a problem with "near-critical" nodes, the NEACRP benchmark problem was modified slightly by removing all control rods and reducing the boron concentration from 561.3 to $389.62 \mathrm{ppm}$. In this case the sixth fuel assembly from the core center on the quarter core axis (assembly A in Fig. 1) becomes very close to critical with the node $k_{\infty}=$ $1.0000041 k_{\text {eff }}$.

When the double-precision version of the code is used, this problem still converges, and the core $k_{\text {eff }}$ is 1.106989 with 4 nodes/FA. Figure 2 shows the individual components of the $x$-directional intranodal thermal flux variation in the vicinity of the near-critical node at the midplane, which were obtained from the double-precision calculation. The fluxes shown here were normalized such that the core average total flux is unity. In part (a) of the figure, which shows the particular solution and fundamentalmode part of the homogeneous solution, it is evident that the particular and fundamental mode solutions are large in magnitude $\left(10^{8}\right)$ and of opposite sign for the near-critical nodes. However, because the large positive and negative terms cancel, the sum of the particular and fundamental solutions becomes the same order of magnitude as the total flux. The magnitude of the harmonic part of the homogeneous solution is one order smaller than that of the sum of the two parts and determines the boundary effects, as shown in part (b) of the figure. The sum of all three components yields the total flux, which is smoothly varying over the nodes.

The large magnitude of the particular solution in nearcritical nodes is because the coefficient matrix $\mathbf{A}_{\infty}$ appearing in Eq. (28) is near singular and highly ill-conditioned. The determinant of $\mathbf{A}_{\infty}$ can be expressed in terms of $\lambda k_{\infty}$ as

$$
\operatorname{Det}\left(\mathbf{A}_{\infty}\right)=\Sigma_{r 1} \Sigma_{r 2}\left(1-\lambda k_{\infty}\right) .
$$

In this particular case, $1-\lambda k_{\infty}=4.1 \times 10^{-6}$, which would lead to an amplification of the $b_{g p}$ coefficients by a factor of $2.5 \times 10^{5}$ for the particular solution given by Eq. (30). In fact, the constant term in the particular solution is determined after being amplified twice because $b_{g 0}$ defined in Eq. (29) contains the second-order coefficient $c_{g 2}$. Therefore, the amplification is roughly proportional to the square of the inverse of $1-\lambda k_{\infty}$. The smaller the value of $1-\lambda k_{\infty}$ is, the greater the magnitude of the particular solution. With the value $4.1 \times 10^{-6}$, the particular solution of this node is on the order of $10^{8}$.

The large magnitude of the particular solution should be properly compensated by the fundamental parts of the

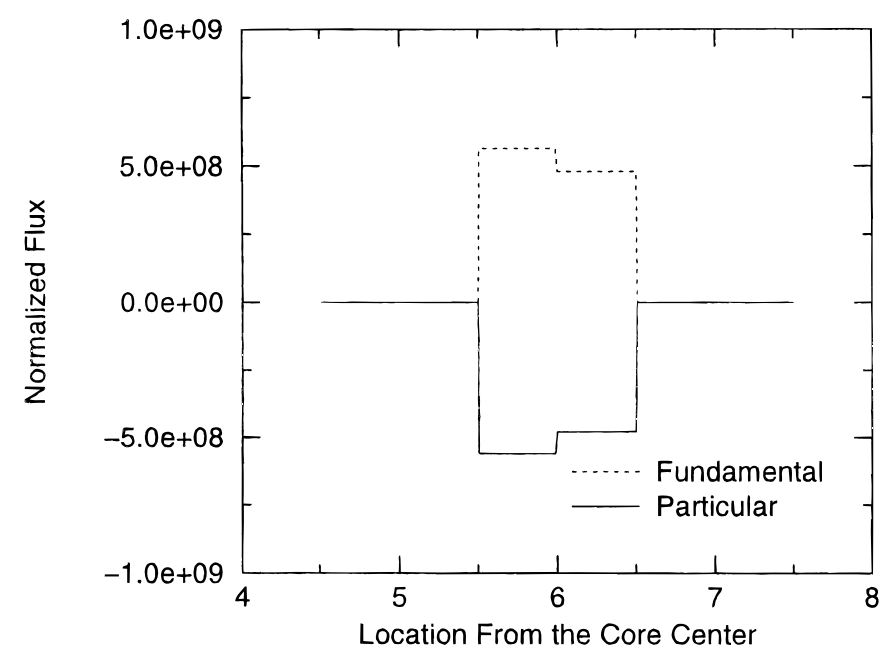

b

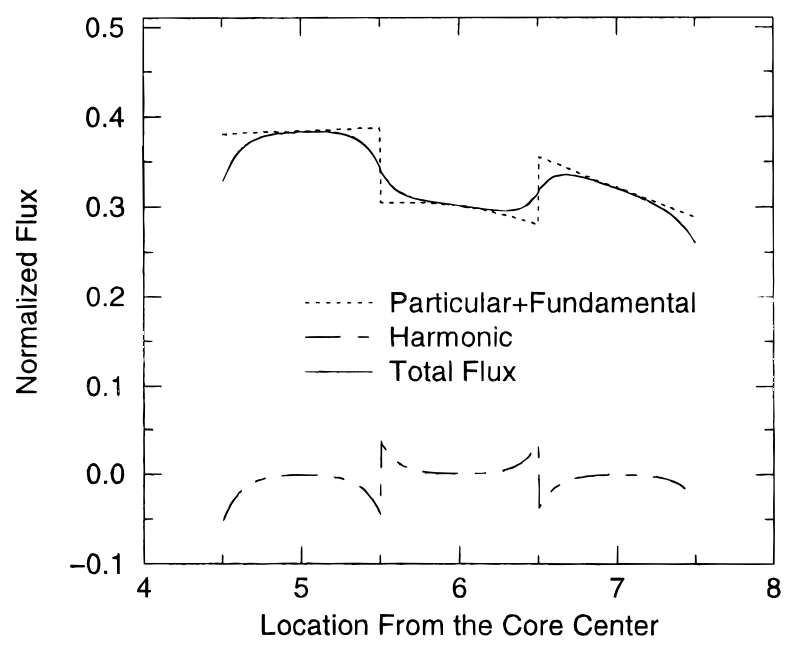

Fig. 2. Components of intranodal flux distribution in the vicinity of a critical node. 
homogeneous solution to yield a flux on the order of unity. An insufficient number of significant digits in singleprecision arithmetic, however, can cause an incomplete compensation, which, in turn, will lead to an incorrect solution. In fact, the single-precision version of the PARCS code did not converge in this case.

The ill-conditioning problem appears not only in finding the particular solution but also in finding the homogeneous solution. The $4 \times 4$ matrices appearing in Eq. (45) become ill-conditioned because the entries in an entire column approach zero as the values of $\kappa$ and $\operatorname{sn}\left(\kappa_{1 / 2}\right)$ approach zero in near-critical nodes.

This analysis indicates that the nature of the ANM solution, in which a large mismatch in the homogeneous and particular solutions exists for near-critical nodes, will exacerbate round-off errors encountered in single-precision arithmetic. Because it is desirable to retain the option of single-precision arithmetic for practical calculations, various techniques have been proposed for stabilizing the ANM solution. The simplest of these was to move a small fraction of the fission source to the right side to eliminate the ill-conditioning of the linear systems, and this approach has apparently been used in some cases. ${ }^{10}$ However, more rigorous approaches are examined here that include first implementing the critical node ANM solution and then employing a hybrid ANM/NEM method in which the NEM two-node solution is used only for near-critical nodes.

\section{STABILIZATION TECHNIQUES}

In this section, alternate techniques are described for stabilizing the ANM solution. First, the ANM critical node solution described in Sec. II.A.2.a is applied to nearcritical nodes as an approximation. A hybrid technique that invokes NEM for near-critical nodes is then described. Consistent comparisons of the methods are performed using the modified NEACRP benchmark problem.

\section{IV.A. Linear Fundamental Mode Approximation}

The ANM solution for a critical node is characterized by a quartic particular solution and by a linear fundamental mode in the homogeneous solution. This solution is exact if and only if the node is critical. It is, however, possible to apply the critical node solution to near-critical nodes to avoid the ill-conditioning problem. In this case, the sin (or sinh) and cos (or cosh) functions in Eq. (13) are approximated by $x$ and 1, respectively, and there would be error in the solution associated with the approximation. In this regard, this approximation will be referred to as the linear fundamental mode (LFM) approximation.

In this approximation, the ANM critical node kernel is activated with a prespecified criterion $\epsilon$. Prior to the two-node calculations, the condition is applied to each node:

$$
\delta \equiv\left|\frac{k_{\infty}}{k_{e f f}}-1\right|<\epsilon
$$

and if satisfied the critical node kernel is applied. Otherwise, the normal kernel is used.

The single-precision solutions of the modified benchmark problem with the critical node kernel are compared with the reference double-precision ANM solution in Table II for 4 nodes/FA. As indicated, if $\epsilon<10^{-6}$, the single-precision solution diverges because the critical ANM kernel is not activated in any node. In case A2, a tolerance of $\epsilon=10^{-3}$ identifies 60 critical nodes, and the solution converges to within $0.2 \mathrm{pcm}$ (per centenas millias $=10^{-5}$ ) of the reference.

The intranodal thermal flux variation obtained from the LFM approximation in case A2 is shown in Fig. 3 in the vicinity of the same critical node to which Fig. 2 applies. In comparison with Fig. 2, it is first noted that neither the particular solution nor the fundamental mode homogeneous solution is large in magnitude. In fact, they

TABLE II

Performance of ANM with Critical Node Kernel for Different Criteria of Near Criticality

\begin{tabular}{|l|c|c|c|r|}
\hline Case & Criterion & Number of Critical Nodes & $k_{\text {eff }}$ Error $^{\mathrm{b}}\left(\mathrm{pcm}, \times 10^{-5}\right)$ & $F_{q}$ Error $^{\mathrm{c}}(\%)$ \\
\hline A1 & $1.0 \times 10^{-6}$ & 0 & Diverges & Diverges \\
A2 & $1.0 \times 10^{-3}$ & 60 & -0.11 & 0.005 \\
A3 & 0.01 & 60 & -0.11 & 0.005 \\
A4 & 0.02 & 180 & 0.08 & -0.021 \\
A5 & 0.04 & 780 & 8.66 & 0.480 \\
A6 & 0.10 & 1868 & 115.72 & 2.535 \\
A7 & 1.00 & 3978 & Diverges & Diverges \\
\hline
\end{tabular}

${ }^{\mathrm{a}}$ Total number of nodes $=3978$.

${ }^{\mathrm{b}}$ Reference: $k_{\text {eff }}=1.1069899$.

${ }^{\mathrm{c}}$ Reference: $F_{q}=1.8976$. 
a

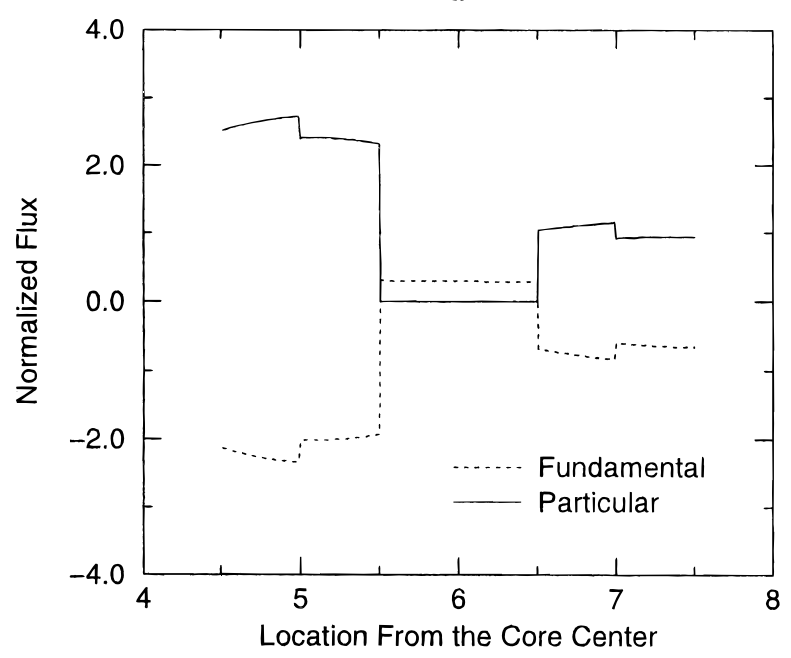

b

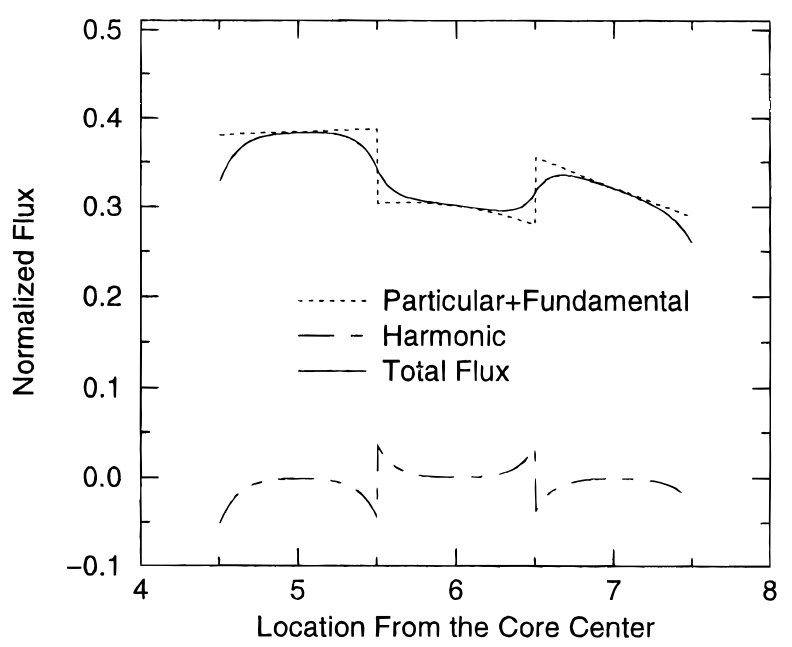

Fig. 3. Components of intranodal flux distribution in the vicinity of a critical node with LFM.

are now smaller than those at the adjacent nodes. It is also noted that each component shown in part (b) of the figure is essentially the same as the corresponding one in Fig. 2b. The stabilization of the particular solution is the primary reason the single-precision calculation with the critical-node kernel converges with negligible errors. The reason for the stabilization is, of course, that there is no ill-conditioned matrix appearing in the determination of the five polynomial coefficients in the particular solution as given in Eq. (38). Ill-conditioning problems also disappear in the $4 \times 4$ matrices appearing in Eq. (45) because the entries no longer approach zero in the LFM approximation.

The sensitivity of the single-precision solution to the value of $\epsilon$ was also examined as shown in cases A3 through A7 in Table II. As the value of $\epsilon$ is increased, thereby relaxing the criterion for criticality, more twonode solutions are performed with the critical-node kernel. This leads to an increase in the error of both the $k_{\text {eff }}$ and the power distribution because the LFM approximation becomes worse as the degree of criticality is relaxed. The solution eventually diverges in case A7 when the value of the critical criterion is 1.0 , at which all the two-node problems are treated with the critical-node kernel.

The error in the intranodal thermal flux distribution due to the LFM approximation is depicted in Fig. 4 for two assemblies (marked A and B in Fig. 1) with different degrees of near criticality (case A4). The intranodal flux distribution was defined as the overlapping of these two two-node solutions. The solid line in the figure is for the same node as shown in Figs. 2 and 3, and the degree of near-criticality is $4.1 \times 10^{-6}$. The dotted line is for a node that has a degree of near criticality of $1.5 \times 10^{-2}$, for which the LFM approximation applies. The reference intranodal flux distribution was taken from the doubleprecision calculation. At location $\mathrm{A}$, the largest intranodal flux error is $0.04 \%$, which is considered small. At location B, however, the intranodal flux error is much larger than at location $\mathrm{A}$, and the largest error is $0.3 \%$. It is also noted that the errors in the neighboring nodes are not negligible near location B. Obviously this is because the LFM approximation becomes worse as the degree of near criticality increases. Therefore, the switching criterion $(\epsilon)$ must not be set too large in LFM approximation.

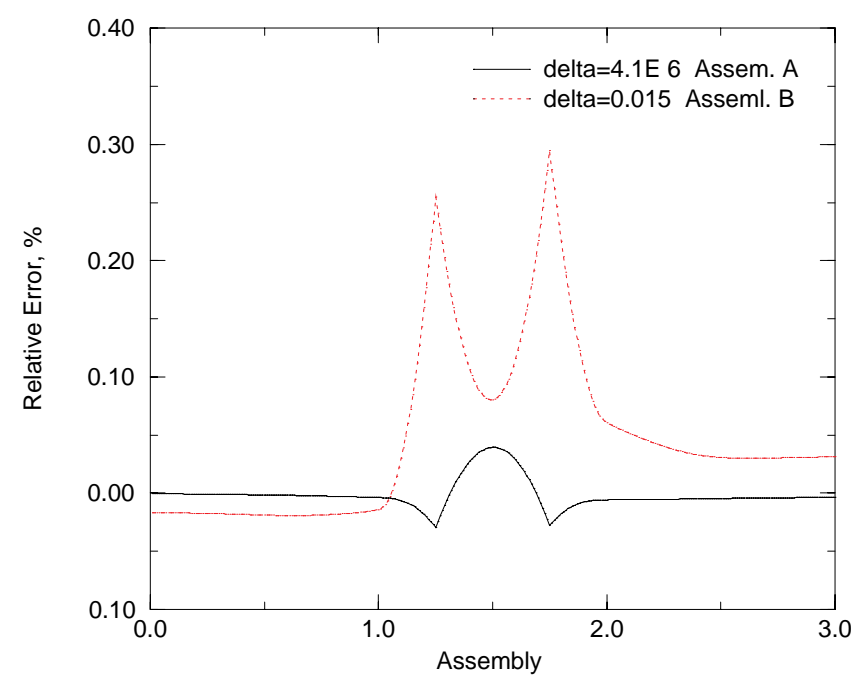

Fig. 4. Relative error of intranodal flux distribution in the vicinity of a critical node with LFM. 
IV.B. Hybrid Analytic Nodal Method/Nodal

Expansion Method Interface Coupling Technique

The derivation of a two-node NEM coupling kernel appears in several references. ${ }^{7}$ In addition to constraints similar to those of the ANM kernel, two moment balance equations are applied to determine the polynomial expansion coefficients. Since the NEM kernel involves no particular solution, it does not have the numerical instability of the ANM. In fact, it is numerically stable under all conditions. Noting the stability of the NEM kernel, a hybrid scheme is examined in which the NEM two-node solution is used for any two-node problem involving a critical node.

The implementation of a hybrid ANM/NEM method is conceptually very simple. The same criterion, Eq. (54), is used to identify near-critical nodes and activate the NEM solution. If the value of $\epsilon$ is greater than unity in this hybrid scheme, the solution of all two-node problems will be based on NEM. On the other hand, if the value of $\epsilon$ is zero, the solution will be based purely on ANM. For the intermediate values of $\epsilon$, both the ANM and NEM are used in different portions of the core.

Results are shown in Table III for single-precision hybrid ANM/NEM calculations for the modified NEACRP problem with a nodalization of 4 nodes/FA. The first case shown in the table is the single-precision solution with pure ANM, which diverges. As in the critical ANM kernel cases shown in Table II, a criterion of $\epsilon=$ $10^{-3}$ identifies 60 near-critical nodes, and the solution again converges to within $0.1 \mathrm{pcm}$ of the reference. However, as shown in cases B3 to B10, the hybrid solution does not deteriorate as rapidly as the criterion increases and converges when $\epsilon=1.00$. In this case the solution becomes purely NEM, and the error in the $k_{\text {eff }}$ is $\sim 15 \mathrm{pcm}$, which is

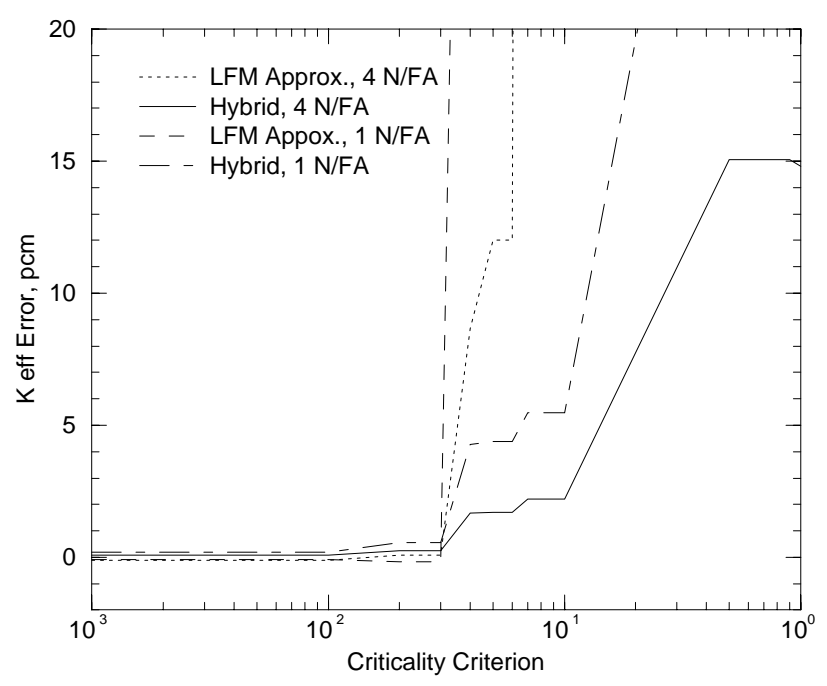

Fig. 5. Sensitivity of ANM solutions to the criticality criterion.

similar to the difference in ANM and NEM as shown in Table I for the original NEACRP case A1.

The accuracy of the hybrid and LFM approximation solutions for various criterion values is compared in Fig. 5 for the 4 node/FA case as well as for the 1 node/ FA case. As indicated in the figure, the accuracy of both methods is comparable for small values of the criterion, regardless of the node size. However, the accuracy of both approximations deteriorates rapidly as the criterion increases beyond 0.01, particularly with the LFM approximation. Therefore, it is recommended that the switching criterion be limited to $<0.01$.

TABLE III

Performance of Hybrid ANM/NEM Method for Different Criteria of Near Criticality

\begin{tabular}{|l|c|c|c|c|}
\hline Case & Criterion & Number of Critical Nodes ${ }^{\mathrm{a}}$ & $k_{\text {eff }}$ Error $^{\mathrm{b}}\left(\mathrm{pcm}, \times 10^{-5}\right)$ & $F_{q}$ Error $^{\mathrm{c}}(\%)$ \\
\hline B1 & $1.0 \times 10^{-6}$ & 0 & Diverges & Diverges \\
B2 & $1.0 \times 10^{-5}$ & 60 & 0.08 & 0.000 \\
B3 & 0.01 & 60 & 0.08 & 0.000 \\
B4 & 0.02 & 180 & 0.25 & -0.005 \\
B5 & 0.04 & 780 & 1.67 & 0.058 \\
B6 & 0.06 & 900 & 1.70 & 0.058 \\
B7 & 0.07 & 1748 & 2.21 & 0.074 \\
B8 & 0.10 & 1868 & 2.21 & 0.074 \\
B9 & 0.90 & 2512 & 15.06 & -0.364 \\
B10 & 1.00 & 3978 & 14.79 & -0.353 \\
\hline
\end{tabular}

${ }^{\mathrm{a}}$ Total number of nodes $=3978$.

${ }^{\mathrm{b}}$ Reference: $k_{\text {eff }}=1.1069899$.

${ }^{\mathrm{c}}$ Reference: $F_{q}=1.8976$. 
It should also be noted that when feedback is applied to the neutronics calculation (for example, thermal hydraulics, xenon, etc.), the number of critical nodes could potentially oscillate during the course of the feedback iteration. However, very simple logic can be applied to detect an oscillation, and the critical nodes can be fixed during the feedback iteration with negligible loss of accuracy.

\section{SUMMARY AND CONCLUSIONS}

The objective of the work here was to develop stabilization techniques for the nonlinear ANM nodal solution. The two-node ANM kernel was derived, and two stabilization techniques were introduced that employ alternate basis functions for critical nodes. The first uses the exact ANM critical node kernel solution for a criticalnode (LFM approximation), and the second employs the NEM. Both techniques were shown to perform well, and the solution accuracy did not change essentially as long as the criterion used to invoke the stabilized coupling kernel was kept $<0.01$.

The work here provides a practical method for preserving the accuracy of the ANM solution for singleprecision calculations. Although, the conditions under which a critical node may be encountered are infrequent, the stabilization techniques proposed here should be considered for implementation of the nonlinear ANM since there are several applications where divergence of the nonlinear nodal solution cannot be tolerated.

\section{ACKNOWLEDGMENT}

The authors wish to express their gratitude to Prof. P. J. Turinsky of North Carolina State University for several valuable discussions during the course of this work.

\section{REFERENCES}

1. K. S. SMITH, "Nodal Method Storage Reduction by Nonlinear Iteration,” Trans. Am. Nucl. Soc., 44, 265 (1983).

2. H. G. JOO and T. J. DOWNAR, "An Incomplete Domain Decomposition Preconditioning Method for Nonlinear Nodal Kinetics Calculations," Nucl. Sci. Eng., 123, 403 (1996).

3. R. LAWRENCE, "Progress in Nodal Methods for the Solution of the Neutron Diffusion and Transport Equations," Prog. Nucl. En., 17, 271 (1986).

4. H. FINNEMANN, F. BENNEWITZ, and M. R. WAGNER, "Interface Current Techniques for Multidimensional Reactor Calculations," Atomkernenergie, 30, 123 (1977).
5. K. SMITH, "An Analytic Nodal Method for Solving the 2-Group, Multi-Dimensional, Static, and Transient Neutron Diffusion Equations," Thesis, Department of Nuclear Engineering, Massachusetts Institute of Technology (1979).

6. T. J. DOWNAR, H. G. JOO, and G. JIANG, "A Hybrid ANM/NEM Interface Current Technique for the Nonlinear Nodal Calculation," Proc. Joint Int. Conf. Mathematical Methods and Supercomputing for Nuclear Applications, Saratoga Springs, New York, October 5-9, 1997, Vol. 1, p. 124, American Nuclear Society (1997).

7. P. TURINSKY et al., "NESTLE: A Few-Group Neutron Diffusion Equation Solver," Electric Power Research Center, North Carolina State University (1994).

8. B. N. AVILES, "Development of a Variable Time-Step Transient NEM Code: SPANDEX," Trans. Am. Nucl. Soc., 68, 425 (1993).

9. K. SMITH and J. T. CRONIN, "SIMULATE-3 Methodology," SOA-92/02, Studsvik of America (1992).

10. R. DEVENEY, "Noodle Code Manual," FCF, Personal Communication (Feb. 1997).

11. T. SUTTON and B. AVILES, "Diffusion Theory Methods for Spatial Kinetics Calculations," Prog. Nucl. Ener., 30, 119 (1996).

12. L. D. EISENHART, "ARROTTA-01: Advanced Rapid Reactor Operational Transient Analysis," EPRI Code Manual, project 1936-6, Electric Power Research Institute (1993).

13. S. NAKAMURA, Computational Methods in Engineering and Science, John Wiley \& Sons, New York (1977).

14. H. G. JOO, T. J. DOWNAR, and D. A. BARBER, "Methods and Performance of a Parallel Reactor Kinetics Code PARCS," Proc. Int. Conf. Physics of Reactors, Mito, Japan, September 16-20, 1996, p. J-42.

15. H. FINNEMANN and A. GALATI, "NEACRP 3D LWR Core Transient Benchmark-Final Specifications," NEACRPL-335 (Rev. 1), Nuclear Energy Agency Committee on Reactor Physics (Jan. 1992).

16. H. G. JOO et al., "Parallel Computing Methods for the EPRI Spatial Kinetics Code ARROTTA," Proc. Topl. Mtg. Advances in Nuclear Fuel Management, II, Myrtle Beach, South Carolina, March 23-26, 1997, p. 20.31, Electric Power Research Institute (1997).

17. M. P. KNIGHT and P. BRYCE, "Derivation of a Refined PANTHER Solution to the NEACRP PWR Rod-Ejection Transients," Proc. Joint Int. Conf. Mathematical Methods and Supercomputing for Nuclear Applications, Saratoga Springs, New York, October 5-9, 1997, Vol. 1, p. 302, American Nuclear Society (1997). 